\title{
ESTUDIO DE LA CONSERVACIÓN DEL GLOBO OCULAR CON UN ABORDAJE MULTITERAPÉUTICO EN EL MELANOMA UVEAL
}

\section{OCULAR RETENTION IN PATIENTS WITH UVEAL MELANOMA MANAGED BY A MULTIMODALITY APPROACH}

\author{
GARCÍA-ÁLVAREZ C ${ }^{1}$, MUIÑOS Y ${ }^{1}$, SAORNIL MA², ALMARAZ A², LÓPEZ-LARA F², \\ FRUTOS JM ${ }^{3}$, MUÑOZ MF ${ }^{4}$
}

\begin{abstract}
RESUMEN
ABSTRACT

Objetivo: Analizar la supervivencia de los globos oculares afectados de melanoma uveal sometidos a tratamiento conservador, en un centro que aplica todas las modalidades de tratamiento.

Métodos: Se han incluido en el estudio los pacientes diagnosticados de melanoma de úvea tratados entre septiembre de 1990 y abril de 2007 en la unidad de Oncología Ocular del Hospital Clínico Universitario de Valladolid realizándose un estudio de cohortes histórico.

Resultados: De los 273 pacientes incluidos en el estudio, se trataron 193 pacientes con terapias conservadoras $(70,69 \%)$, mientras que se enuclearon de forma primaria $80(29,30 \%)$. Se enuclearon de forma secundaria 14 de los sometidos a tratamiento conservador $(7,2 \%) \mathrm{El}$ análisis de supervivencia de Kaplan-Meier reveló que tras la aplicación de un tratamiento conservador, la probabilidad de conservación del globo ocular a los 5 años es del $88 \%$ y a los 10 años es del 83\%.

Conclusiones: El presente estudio pone de manifiesto la seguridad de los tratamientos conservado-

Objective: to analyze ocular survival in eyes with uveal melanoma treated with conservative therapies, in a centre that applies all treatment modalities.

Methods: Patients diagnosed with uveal melanoma and treated between September 1990 and April 2007 were included in an historical cohorts study. Results: 273 patients were included. 193 were treated with conservative treatments $(70.69 \%)$ and 80 were enucleated as primary treatment. 14 patients were enucleated after conservative treatment (7.2\%). Kaplan-Meier survival analysis showed an $88 \%$ survival probability of the eye in the first 5 years after conservative treatment and $83 \%$ at 10 years.

Conclusions: Conservative treatments for uveal melanoma, especially brachitherapy, are safe and effective in relation to tumor control and rate of secondary effects (Arch Soc Esp Oftalmol 2009; 84: 145-150).

Key words: Intraocular tumors, uveal melanoma, episcleral brachitherapy, enucleation.

\footnotetext{
Recibido: 25/6/08. Aceptado: 23/3/09.

Unidad de Tumores Intraoculares del Adulto. CSUR del SNS. Servicios de Radioterapia, Oftalmología y Unidad de Investigación. Hospital

Clínico Universitario. Valladolid. España.

${ }^{1}$ Licenciado en Medicina.

${ }^{2}$ Doctor en Medicina.

3 Licenciado de Física.

${ }^{4}$ Licenciado en Estadística.

Correspondencia:

Ciro García-Álvarez

Hospital Clínico Universitario

Ramón y Cajal

47003 Valladolid

España

E-mail: cigore@yahoo.es
} 
res en el melanoma uveal, especialmente de la braquiterapia epiescleral, en cuanto a control local de la enfermedad y a tasa de efectos secundarios.

Palabras clave: Tumores intraoculares, melanoma uveal, braquiterapia epiescleral, enucleación.

\section{INTRODUCCIÓN}

El melanoma uveal es la neoplasia ocular primaria más frecuente en el adulto con una incidencia estimada en Estados Unidos de aproximadamente 5 casos por millón de habitantes y año (1), y desconocida en nuestro medio.

El tratamiento de esta enfermedad se basó durante mucho tiempo en la enucleación, pero en las últimas décadas del pasado siglo se desarrollaron técnicas conservadoras que han demostrado ser tan eficaces como la primera en cuanto a control local en tumores de determinadas características (2). Estos tratamientos locales además de ser menos mutilantes, permiten conservar el globo ocular y mantener la función visual de los pacientes (3-8). En cuanto al control sistémico de la enfermedad, ni las terapias conservadoras ni la enucleación han demostrado ser superiores en cuanto a disminuir la incidencia de metástasis en el seguimiento de los pacientes (9).

Estas técnicas conservadoras son la braquiterapia epiescleral con distintos isótopos (los más empleados son el Rutenio 106 y el Yodo 125), la radioterapia externa con protones, la termoterapia transpupilar (si bien hoy en día se ha abandonado como tratamiento en exclusiva y se emplea asociada a los otros) (10) y la endo y exoresección quirúrgica de los tumores. El fracaso en el control de la enfermedad (aparición de recurrencias) o la aparición de efectos secundarios una vez aplicados estos tratamientos hacen a veces necesaria la enucleación de forma secundaria de los ojos previamente tratados.

El objetivo de este trabajo es analizar la supervivencia de los globos oculares afectados de melanoma uveal sometido a tratamiento conservador, en un centro que aplica todas las modalidades de tratamiento.

\section{SUJETOS, MATERIAL Y MÉTODOS}

Se han incluido en el estudio prospectiva y consecutivamente los pacientes diagnosticados de melanoma de coroides tratados entre septiembre de 1990 y abril de 2007 en la unidad de Oncología Ocular del Hospital Clínico Universitario de Valladolid.

En todos los pacientes se realizó una exploración ocular completa incluyendo determinación de la mejor agudeza visual (AV) con corrección óptica, biomicroscopía de polo anterior, examen de fondo de ojo con retinografía y ecografía $\mathrm{B}$ con vector $\mathrm{A}$, realizando medidas del tumor. Se utilizaron otras técnicas de imagen como resonancia magnética (RM) y/o Tomografía axial computerizada (TAC) en caso de opacidades de medios y/o desprendimiento de retina extenso que impedían evaluar correctamente el tamaño del tumor, y también para valorar la existencia de extensión extraocular. Para la evaluación de la extensión sistémica se practicaron ecografías hepáticas y perfiles hepáticos sanguíneos.

El criterio de diagnóstico de melanoma se ha basado en observar una lesión de aspecto oftalmoscópico y características ecográficas compatibles con una altura mayor de $3 \mathrm{~mm}$ o base mayor de 5 $\mathrm{mm}$. Basándonos en los criterios publicados por el «Collaborative Ocular Melanoma Study» $(5,7)$, se han definido como melanomas de tamaño pequeño los tumores con altura menor o igual a $3,1 \mathrm{~mm}$ y base entre 5 y $10 \mathrm{~mm}$; medianos aquellos con altura entre 3,1 y $10 \mathrm{~mm}$ y base menor o igual a $16 \mathrm{~mm}$, y de tamaño grande aquellos con más de $10,1 \mathrm{~mm}$ de altura o mas de $16 \mathrm{~mm}$ de base. Los signos de actividad se han basado en los descritos por Shields (10) y han sido la presencia de pigmento naranja, fluido subretiniano (desprendimiento de retina asociado), forma de champiñón o crecimiento documentado (5) así como la presencia de drusas se ha considerado signo de falta de actividad.

Se registraron los datos de filiación del paciente, sus antecedentes generales, antecedentes oftalmológicos, datos clínicos (fecha de inicio de los síntomas, fecha de diagnóstico y motivo del diagnóstico), exploración (lateralidad, agudeza visual, presión intraocular, color del iris, invasión del iris, rubeosis, afectación del cristalino, hemovítreo, invasión angu- 
lar y otras), localización y tamaño (base máxima, base a 90 grados, altura máxima, distancia a nervio óptico, distancia a fóvea, localización del borde anterior, localización del borde posterior, localización anterior o posterior, localización temporal o nasal, número de cuadrantes afectados, afectación macular y forma del tumor: plano, nodular o champiñón), Signos de actividad, causas de disminución de agudeza visual por melanoma (catarata, glaucoma, hemovítreo, desprendimiento de retina, hemorragia subretiniana, edema de mácula o tumor macular), pruebas de extensión extraocular, pruebas de extensión sistémica, clasificación del melanoma, tratamientos y presencia de metástasis.

El cuestionario se completó a partir de preguntas formuladas al propio paciente, la exploración y las pruebas complementarias realizadas. El encargado de recoger los datos en los cuestionarios fue un oftalmólogo (adjunto, residente o alumno master) perteneciente a la unidad. Una vez completado el cuestionario, se codificó en una base de datos diseñada en Microsoft ${ }^{\circledR}$ Acces $^{\circledR}$ previo consentimiento informado del paciente. El encargado de codificar los datos fue también un oftalmólogo (residente, alumno master o adjunto) perteneciente a la unidad.

\section{Protocolo de tratamiento}

La braquiterapia se indicó en los tumores de tamaño mediano, o de tamaño pequeño con crecimiento documentado. También se indicó en tumores grandes en los que el paciente rechazó la enucleación o en casos de ojo único afectado.

Se observaron los tumores clasificados como pequeños que no presentaban signos de actividad.

La radioterapia externa con protones se indicó en tumores cuya cercanía al nervio óptico desaconsejaba el uso de radioterapia epiescleral o con un tamaño grande (altura $>10 \mathrm{~mm}$ y/o base $>16 \mathrm{~mm}$ ) en los que se decidió aplicar terapia conservadora.

La termoterapia transpupilar se utilizó únicamente como tratamiento adyuvante de los anteriores.

La extirpación quirúrgica se indicó en pacientes con melanomas medianos preecuatoriales no subsidiarios de tratamiento con braquiterapia.

Se enuclearon los pacientes que presentaban tumores grandes, función visual irrecuperable, invasión del nervio óptico, extensión extraocular, tumores planos infiltrativos difusos o si el paciente por motivos psicológicos rehusó el tratamiento conservador.

\section{Evaluación de resultados y seguimiento}

Los pacientes fueron revisados en la unidad de Oncología Ocular cada 6 meses hasta los 5 años y posteriormente de forma anual para valorar la aparición de complicaciones y comprobar la eficacia del tratamiento. En dichas consultas se recogió agudeza visual, biomicroscopía del polo anterior, presión intraocular, fondo de ojo y exploración ecográfica. Se consideró que el tratamiento había tenido éxito si el tumor había dejado de crecer en los sucesivos controles ecográficos o había disminuido de tamaño (11). En las revisiones se solicitó ecografía hepática y perfil analítico hepático para descartar extensión sistémica de la enfermedad.

\section{Tratamiento estadístico}

Se ha realizado un estudio de cohortes histórico, los datos aparecen representados en tablas de frecuencias. La probabilidad de supervivencia de los globos oculares se calculó mediante el análisis de supervivencia de Kaplan-Meier. Aquellos valores de $\mathrm{p}<0,05$ han sido considerados estadísticamente significativos. Para el análisis estadístico se ha utilizado el programa estadístico SPSS ${ }^{\text {TM }}$ Versión 12.0 (SPSS Inc, Chicago, Illinois, USA).

\section{RESULTADOS}

\section{Perfil epidemiológico y tumoral de los pacientes incluidos en el estudio}

Se incluyeron en el estudio 273 pacientes diagnosticados de melanoma uveal y tratados u observados entre enero de 1990 y abril de 2007.

La edad media fue de 61,48 años (desviación estándar: 14,64; Mínimo: 16; Máximo 93). No se observaron diferencias en cuanto a sexo ni lateralidad (tabla I). El seguimiento medio fue de 53,46 meses (desviación estándar: 37,73; mínimo: 27; máximo 117).

\section{Tratamiento primario}

Se trataron 193 pacientes con terapias conservadoras $(70,69 \%)$, mientras que se enuclearon de forma primaria $80(29,30 \%)$. En la tabla II se pueden 
Tabla I.

\begin{tabular}{lcc}
\hline & $\mathrm{n}$ & $\%$ \\
\hline Sexo & & \\
Hombre & 127 & 46,52 \\
Mujer & 146 & 53,47 \\
Lateralidad & & \\
OD & 150 & 54,94 \\
OI & 122 & 44,65 \\
\hline \hline
\end{tabular}

Tabla II.

\begin{tabular}{lr}
\hline Tratamiento & $\mathrm{n}$ \\
\hline Enucleación & 80 \\
Cirugía & 4 \\
TTT & 14 \\
RT con protones & 11 \\
Braquiterapia & 121 \\
Observación & 43 \\
Total & 273 \\
\hline \hline
\end{tabular}

observar estos datos desglosados según el tratamiento aplicado.

\section{Enucleación secundaria}

Se enuclearon de forma secundaria aquellos pacientes en los que el tratamiento fracasó o en los que aparecieron efectos secundarios que no respondieron a ningún tratamiento. Del total de 193 tratados, 14 (el 7,2\%) fueron enucleados de forma secundaria. En la tabla III puede verse el porcentaje de enucleaciones secundarias en función del tratamiento (tabla III).

El análisis de supervivencia de Kaplan-Meier (fig. 1) reveló que tras la aplicación de un tratamiento conservador, la probabilidad de conservación del globo ocular a los 5 años es del $88 \%$ y a los 10 años es del $83 \%$.

Tabla III

\begin{tabular}{lrc}
\hline Tratamiento & $\mathrm{n}$ & Enucleación 2. ${ }^{\mathrm{a}}(\%)$ \\
\hline Cirugía & 4 & $1(25 \%)$ \\
TTT & 14 & $1(7,1 \%)$ \\
RT con protones & 11 & $6(54,5 \%)$ \\
Braquiterapia & 121 & $6(4,9 \%)$ \\
Observación & 43 & 0 \\
Total & 193 & $14 \quad(7,2 \%)$ \\
\hline \hline
\end{tabular}

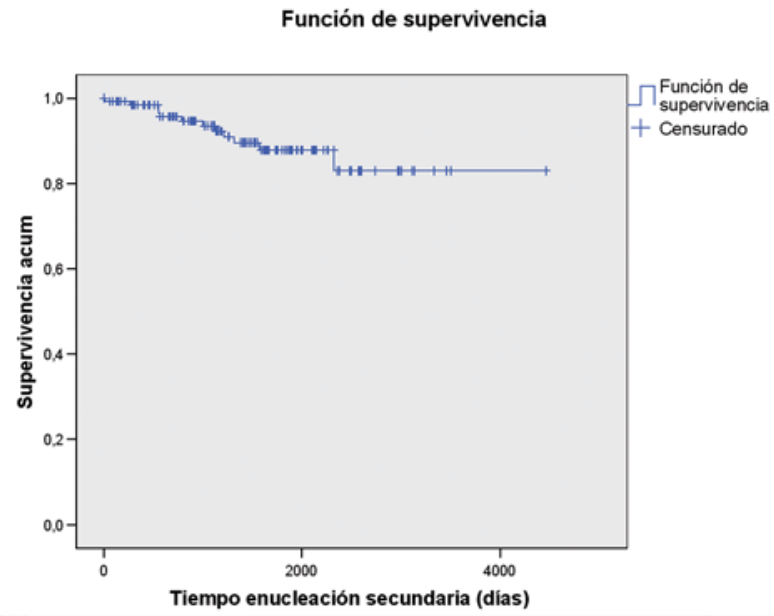

Fig. 1 .

\section{DISCUSIÓN}

En la serie estudiada, de 273 pacientes diagnosticados de melanoma uveal, 80 (29,3\%) (tabla I) requirieron enucleación de entrada, en su mayoría por tratarse de tumores grandes. El diagnóstico precoz de esta enfermedad es de vital importancia, puesto que permite el empleo de terapias conservadoras. Estos tratamientos conservadores han demostrado ser tan eficaces como la enucleación en cuanto al control local de la enfermedad siendo menos mutilantes y permitiendo preservar la función visual en muchos casos.

En cuanto a los ojos tratados mediante procedimientos conservadores, de 193 fue necesario enuclear de forma secundaria $14(7,2 \%)$. El tratamiento conservador más empleado fue la braquiterapia epiescleral que además resultó ser la terapia con mejores resultados de forma que de 121 pacientes tratados con esta modalidad de tratamiento, sólo 6 (el 4,9\%) precisó enucleación secundaria. En este punto es importante aclarar que hoy por hoy el empleo de la TTT como tratamiento aislado en el melanoma de coroides es muy controvertido y se recomienda su uso asociado a braquiterapia (11).

El análisis de supervivencia reveló que la probabilidad de supervivencia de un globo ocular tratado mediante tratamiento conservador es del $88 \%$ a los 5 años y del $83 \%$ a los 10 años, mejorándose estos resultados si se aplica sólo a los tratados mediante braquiterapia.

Los resultados del presente estudio son comparables a los publicados por el Collavorative Ocular Melanoma Study Group y por Damato $(12,13)$, en 
Tabla IV.

\begin{tabular}{lrcrc}
\hline Estudio & $\mathrm{n}$ & $\begin{array}{c}\% \\
\text { tratamientos conservadores }\end{array}$ & $\begin{array}{c}\% \\
\text { enucleaciones secundarias }\end{array}$ & $\begin{array}{c}\text { Probabilidad estimada de conservación } \\
\text { del globo ocular a 5 años }\end{array}$ \\
\hline Damat et al & 1.632 & $65 \%$ & $8,15 \%$ & $89 \%$ \\
COMS rerport 19 & 638 & $100 \%$ & $10,81 \%$ & $87,5 \%$ \\
García-Álvarez & 273 & 70,69 & $7,25 \%$ & $88 \%$ \\
\hline \hline
\end{tabular}

cuanto al porcentaje de enucleaciones secundarias y la probabilidad de conservación del globo ocular a los 5 años (tabla IV).

El presente estudio pone de manifiesto la seguridad de los tratamientos conservadores en el melanoma uveal, especialmente de la braquiterapia epiescleral, en cuanto a control local de la enfermedad y a tasa de efectos secundarios. La principal limitación de éstos es el tamaño tumoral, puesto que los tumores grandes están abocados a la enucleación, siendo por lo tanto de vital importancia el diagnóstico precoz.

\section{BIBLIOGRAFÍA}

1. Singh A, Topham A. Incidence of uveal melanoma in the United States: 1973-1997. Ophthalmology 2003; 110: 956-961.

2. Bell JB, Wilson MD. Choroidal Melanoma: Natural History and management options. Cancer Control 2004; 11: 296-303.

3. Diener-West M, Earle JD, Fine SL, Hawkins BS, Moy CS, Reynolds SM, et al. Collaborative Ocular Melanoma Study Group. The COMS randomized trial of iodine 125 brachytherapy for choroidal melanoma: initial mortality findings. COMS Report No. 18. Arch Ophthalmol 2001; 119: 969-982.

4. Seddon JM, Gragoudas ES, Egan KM, Glynn RJ, Howard $S$, Fante $R G$, et al. Relative survival rates after alternative therapies for uveal melanoma. Ophthalmology 1990; 97: 769-777.
5. Augsburger JJ, Schneider S, Freyre J, Brady LW. Survival following enucleation versus plaque radiotherapy in statistically matched subgroups of patients with choroidal melanomas: results in patients treated between 1980 and 1987. Graefes Arch Clin Exp Ophthalmol 1999; 237: 558567.

6. Damato B. Developments in the management of uveal melanoma. Clin Exp Ophthalmol 2004; 32: 639-647.

7. Margo CE. The collaborative ocular melanoma study: an overview. Cancer Control 2004; 11: 304-309.

8. The collaborative Ocular Melanoma Study (COMS) randomized trial of pre-enucleation radiation of large choroidal melanoma: initial mortality findings. COMS report no. 10. Am J Ophthalmol 1998; 125: 779-796.

9. Singh AD, Rennie IG, Kivela T, Seregard S, Grossniklaus H. The Zimmerman-McLean-Foster hypothesis: 25 years later. Br J Ophthalmol 2004; 88: 962-967.

10. Shields CL, Cater J, Shields JA, Singh AD, Santos MC, Carvalho C. Combination of clinical factors predictive of growth of small choroidal melanocytic tumors. Arch Ophthalmol 2000; 118: 360-364.

11. Win PH, Robertson DM, Buettner H, McCannel CA, Bennett SR. Extended follow-up of small melanocytic choroidal tumors treated with transpupillary thermotherapy. Arch Ophthalmol 2006; 124: 503-506.

12. Damato B, Lecuona K. Conservation of eyes with choroidal melanoma by a multimodality approach to treatment. Ophthalmology 2004; 111: 977-983.

13. Jampol LM, Moy CS, Murray TG, Reynolds SM, Albert DM, Schachat AP, et al. The COMS Randomized Trial of Iodine 125 brachytherapy for Choroidal Melanoma. Local Treatment Failure and Enucleation in the First 5 Years after Brachytherapy. COMS Report No. 19. Ophthalmology 2002; 109: 2197-2206. 
